\title{
Using synthetic oligonucleotides as standards in probe-based qPCR
}

\begin{abstract}
Jillian Conte ${ }^{1,2}$, Margret J Potoczniak ${ }^{3}$ \& Shanan S Tobe ${ }^{3,4}$ ${ }^{1}$ Keystone College, Department of Biological \& Physical Sciences, One College Green, La Plume, PA 18440, USA; '2University of the Sciences, Department of Biological Sciences, Philadelphia, PA, USA; ${ }^{3}$ Arcadia University, Department of Chemistry \& Physics, Glenside, PA, USA; ${ }^{4}$ College of Science \& Engineering, Flinders University, Adelaide, SA 5042, Australia
\end{abstract}

*Author for correspondence: jillian.conte@keystone.edu

BioTechniques 64:177-179 (April 2018) 10.2144/btn-2018-2000

Keywords: DNA standard $\bullet$ gBlocks $^{\circledR} \bullet$ qPCR $\bullet$ synthetic oligonucleotide

Real-time PCR (qPCR) is widely used in the life sciences. For quantifying DNA, a standard curve is required. Common methods for standard development are time consuming, costly, necessitate a specific skill set, and pose a contamination risk. Using a targeted synthetic oligonucleotide, such as a gBlocks ${ }^{\circledR}$ Gene Fragment, overcomes these drawbacks and provides researchers an accurate and quick solution to standard development. Here, we demonstrate that using a gBlocks fragment as a standard provides comparable sensitivity, reliability, and assay performance to a purified amplicon standard.

Since 1992, quantitative PCR (qPCR) has been a cornerstone of molecular genetics. To date, there are over 115,000 and 149,000 citations in PubMed for $\mathrm{QPCR}$ and real-time $\mathrm{PCR}$, respectively. qPCR has a broad range of applications in qualitative and quantitative analyses in diverse fields such as molecular diagnostics, medicine, microbiology, and forensic biology [1-3]. It can be used for simple identification of an amplicon, for absolute or relative quantification, or both. Detection of target amplicons during qPCR is facilitated through either the use of a non-specific DNA-binding dye such as SYBR Green or sequence-specific DNA probes such as TaqMan ${ }^{\circledR}$ probes [4]. When using qPCR for quantification rather than identification, a standard curve is generated from known quantities of the target and is a requirement under the MIQE guidelines and for assessing PCR performance [5].

Commercially available quantification kits are provided with validated standards, but are not cross-compatible with other kits and may not be suitable for use with custom targets [6]. Custom targets such as mitochondrial targets, research for rare diseases, and samples from non-human sources can be difficult to quantify reliably. Current methods for creating custom standard templates include genomic DNA, linear PCR amplicons, or cloned target sequences $[7,8]$. Each method has inherent advantages and disadvantages.
For example, genomic DNA requires a best guess when quantifying $\mathrm{nDNA}$ and guessing a relative mtDNA copy number; PCR amplicons need to be purified and pose a major contamination risk to the laboratory owing to the requirement for manipulating concentrated PCR products; and cloning is inaccurate as plasmid copy quantification per cell is unreliable and requires specialist equipment and training [9,10]. All of these methods can add significant extra costs to assay development. Constructing a synthetic targeted oligonucleotide for use as a standard overcomes all of these drawbacks.

Synthetic oligonucleotides such a gBlocks ${ }^{\circledR}$ Gene Fragments (Integrated DNA Technologies, IA, USA) can be purchased in lengths of 125-3000 bp. This doublestranded product is affordable and easy to obtain, and overcomes all of the disadvantages inherent in other methods for custom standard development. Additionally, this product can provide a qPCR standard for difficult to obtain samples such as those from non-human sources or those including rare mutations. Here, we have incorporated a gBlocks fragment as a DNA standard in a GPCR assay, which decreases time, reagents, and cost of creating a standard sequence.

Primers, a TaqMan probe, and a 126 bp gBlocks fragment were designed and purchased for amplification and detection. For comparison, a non-synthetic standard was also prepared from DNA extracted using the DNA IQ ${ }^{\text {TM }}$ System (Promega Corporation, WI, USA) following the manufacturer's protocol. Primers were used to amplify the target region, which was separated on a 1.8\% agarose gel, excised, and purified with the Qiagen QIAquick gel extraction kit (Qiagen, MD, USA). The non-synthetic standard was quantified using a Qubit (Thermo Fisher Scientific), and subsequently used to compare the efficiency of the gBlocks fragment standard.

The mass of the gBlocks fragment was provided from the manufacturer with purchase and is critical in determining copies present for standard curve development. The gene targeted in this assay

\section{METHOD SUMMARY}

We demonstrate the use of a synthetic oligonucleotide fragment for use as a qPCR standard for DNA quantitation. Comparisons were done with a standard prepared with amplicons. 
is a mitochondrial gene with a commonly accepted estimate of 500 gene copies per cell, which allows for conversion of copy number to concentration [11]. The calculation for copy number in the reconstituted gBlocks fragment was done according to the formula [7]:

(\# bp in $\left.\mathrm{gBlocks}{ }^{\varpi}\right) \times 617.5 \mathrm{~g} / \mathrm{mol} / \mathrm{bp} \times\left(1 \mathrm{~mol} / 6.02 \times 10^{23}\right.$ molecules $)=$ weight per copy

Reconstitution of the lyophilized gBlocks fragment provided over $10^{12}$ copies of the target. Dilution of the stock standard was done in $\mathrm{TE}^{-4}$ buffer to create a sub-stock that was used to prepare the standard curve for the qPCR assay.

Preparation of the standards consisted of diluting the concentrated standard, gBlocks fragment, or purified amplicon to $10^{6}$ copies/ $\mu$, followed by a 1:10 serial dilution to 1 copy/ $\mu$ l. The KAPA Probe Fast qPCR kit (KAPA Biosystems, Inc., MA, USA) was used for amplification and was performed using a Rotor-Gene Q 5plex HRM. Cycling conditions followed the kit protocol. A standard curve was obtained the natural log of concentration (copies/ $\mu \mathrm{l}$ ).

Exponential amplification of both standards was observed and the anticipated Cq increase between serial dilutions of 3.3 was noted (Figure 1 A \& B). No loss of assay efficiency was observed with the gBlocks fragment standard compared to the purified amplicon standard (Figure 1C \& D). No amplification was observed in reactions without template. The sensitivity of both standards was one copy of DNA. single resuspension to generate a stock by plotting the threshold cycle (Cq $)$ versus

Synthetic oligonucleotides require a

standard, whereas linear PCR requires amplification, gel separation, gel excision, gel purification, and DNA quantification to reach the same end point. Each of these steps is a potential contamination risk, not only for the laboratory, but to the standard itself. Relative to the process of creating the standard via linear PCR, the gBlocks fragment was faster, cheaper, simpler and was less of a contamination risk to the laboratory.

Designing the gBlocks fragment is straightforward when using the target sequence as the oligonucleotide sequence. The fragment costs less than US\$100 and arrives within an estimated 2 business days with no laboratory time needed. In comparison, the estimated cost for raw materials needed to prepare a linear PCR amplicon standard is roughly $\$ 50$, and the time required is at least $8 \mathrm{~h}$. The linear PCR amplicon standard preparation necessitates many sample manipulations that expose the laboratory and the standard to contamination. A laboratory contaminating event can cost thousands of dollars to correct and cause a loss of productivity.

One benefit of synthetic oligonucleotides is that a quality control barcode can be incorporated to differentiate the products as synthetic standards or true targets. This is impossible using the other methods that generate exact copies of the target sequence. This approach requires the scientist to have specialized training and experience in strict anti-contamination protocols, whereas the gBlocks fragment standard only requires training in standard operating procedures.

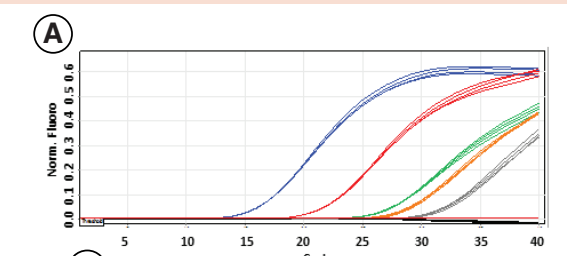

(C)
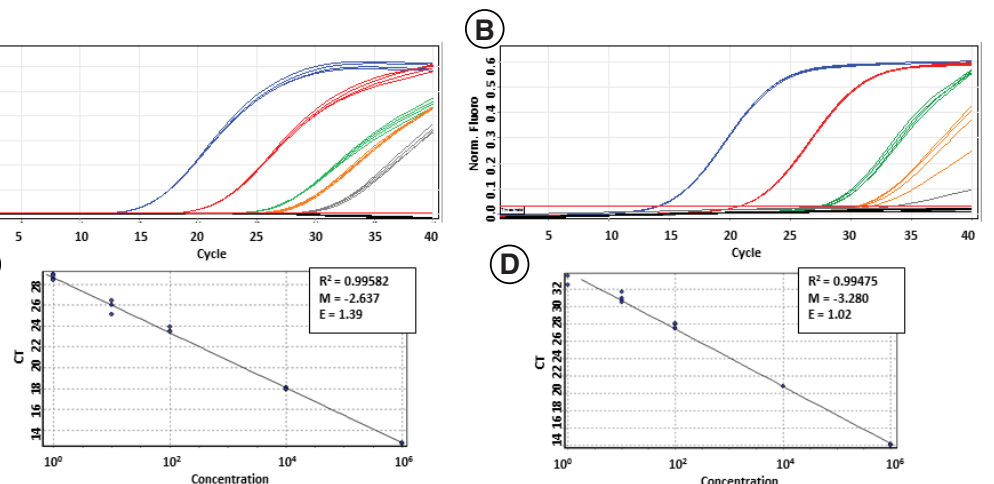

(D)

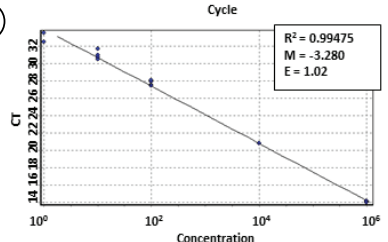

Figure 1. Comparison of qPCR assay efficiency and sensitivity between gBlocks $^{\circledR}$ and purified amplicon standards. The top panels show the exponential amplification of the serially diluted standard, gBlocks ${ }^{\circledR}$ (A) and purified amplicon (B). Blue $=106$ copies, Red $=104$ copies, Green $=102$ copies, Orange = 101 copies, Gray = 1 copy, Black = no template control. The bottom panels show the standard curves generated by the different standards, gBlocks (C) and purified amplicon (D). A high efficiency (greater than $90 \%$ ) and sensitivity (1 copy) was obtained for both using the equation Ex $=10^{\left(-1 / \text { slope }^{-1} \text {. }\right.}$.

\section{Author contributions}

JC and SST designed the study and wrote the manuscript. JC, SST, and MJP conducted the experimental work.

\section{Acknowledgements}

We thank Courtney Mower for assistance with the experimental work. We thank Six Flags Animal Safari, Jackson, NJ, USA for providing buccal swab samples.

\section{Financial \& competing interests disclosure}

This work has been supported by Arcadia University's Masters of Science in Forensic Science program and Keystone College's Department of Biological and Physical Sciences. The authors have no other relevant affiliations or financial involvement with any organization or entity with a financial interest in or financial conflict with the subject matter or materials discussed in the manuscript apart from those disclosed.

No writing assistance was utilized in the production of this manuscript.

\section{References}

1. Bustin SA. Developments in real-time PCR research and molecular diagnostics. Exp. Rev. Mol. Diag. 10(6), 713-715 (2010).

2. Postollec F, Falentin H, Pavan S, Combrisson J, Sohier D. Recent advances in quantitative PCR (qPCR) applications in food microbiology. Food Microbiol. 28(5), 848-861 (2011).

3. Wang Z, Luo H, Pan X, Liao M, Hou Y. A model for data analysis of microRNA expression in forensic body fluid identification. Forensic Sci. Intl: Genetics 6(3), 419-423 (2012).

4. Arya M, Shergill IS, Williamson M, Gommersall L, Arya N, Patel HR. Basic principles of real-time quantitative PCR. Exp. Rev. Mol. Diag. 5(2), 209 219 (2005)

5. Bustin SA, Benes V, Garson JA The MIQE guidelines: minimum information for publication of quantitative real-time PCR experiments. Clin. Chem. 55(4), 611-622 (2009).

6. Nielsen K, Mogensen HS, Eriksen B, Hedman J, Parson W, Morling N. Comparison of six DNA quantification methods. Int. Congress Series 1288(Suppl. C), 759-761 (2006).

7. Goodwin C, Higgins D, Tobe SS, Austin J, Wotherspoon A, Gahan ME, McNevin D. Singleplex quantitative real-time PCR for the assessment of human mitochondrial DNA quantity and quality. Forensic Sci. Med. Pathol. 14(1), 70-75 (2018).

8. Dhanasekaran S, Doherty TM, Kenneth J. Comparison of different standards for real-time PCR-based absolute quantification. J. Immunol. Methods 354(1), 34-39 (2010).

9. Cimino GD, Metchette KC, Tessman JW, Hearst JE, Isaacs ST. Post-PCR sterilization: a method 
to control carryover contamination for the polymerase chain reaction. Nucleic Acids Res. 19(1), 99-107 (1991).

10. Whelan JA, Russell, NB, Whelan MA. A method for the absolute quantification of cDNA using real-time PCR. J. Immunol. Methods 278(1), 261-269 (2003).

11. Satoh M, Kuroiwa T. Organization of multiple nucleoids and DNA molecules in mitochondria of a human cell. Exp. Cell Res. 196(1), 137-140 (1991).

Received 24 January 2018; Accepted for publication: 2 March 2018

Address correspondence to Jillian Conte, Keystone College, Department of Biological \& Physical Sciences, One College Green, La Plume, PA 18440, USA.

E-mail: jillian.conte@keystone.edu

To purchase reprints of this article, contact

s.cavana@future-science.com 